\title{
Relationship between BMI and hip muscle strength in young female athletes to prevent lower extremity injury risk: A pilot study
}

Akshaya M V *1, Abhilash P V ${ }^{2}$, Priya $\mathrm{S}^{3}$.

${ }^{* 1}$ Post graduate student from Laxmi memorial College of physiotherapy, Mangalore, Karnataka, India.

${ }^{2}$ Post graduate student from Laxmi memorial College of physiotherapy, Mangalore, Karnataka, India.

${ }^{3}$ Post graduate student from Laxmi memorial College of physiotherapy, Mangalore, Karnataka, India.

\section{ABSTRACT}

Background: Early identification of the BMI and muscle weakness, can be promoted for developing future rehabilitation by giving proper training in athletes to reduce chance of injuries especially in female athletes. There-for the purpose of this study was to determine the correlation between BMI and hip muscle strength in young female athletes.

Materials and Methods: study was conducted among college level female athletes from different colleges of Mangalore, Karnataka, India. 20 college level female athletes between 18-25 years with free from injury and involved at least $2 \mathrm{hrs}$. per week training session were included in this study. Athletes were excluded if participant had an acute injury during previous six months, had musculoskeletal surgery within the past year.

Results: The total number of 20 young female athletes aged between 18- 25 were included in this study. Detailed results enumerated in detail in the results section.

Discussion and Conclusion: There was no relationship between BMI and hip muscle strength. Identifying the relationship between $\mathrm{BMI}$ and hip muscle strength may help to prevent lower extremity injury risk in female athletes and specific muscle group training can be given as rehabilitation protocol.

KEY WORDS: BMI, Hip Muscle Strength, Female Athletes, Lower Extremity Injury.

Address for correspondence: Akshaya M V, Post graduate student from Laxmi memorial College of physiotherapy, Mangalore, Karnataka, India. E-Mail: akshayaa51@gmail.com

Access this Article online

Quick Response code

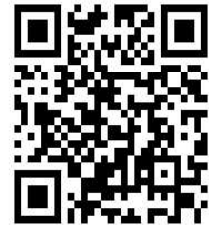

DOI: $10.16965 /$ ijpr.2020.190
Journal Information

International Journal of Physiotherapy and Research

ISSN (E) 2321-1822 | ISSN (P) 2321-8975

https://www.ijmhr.org/ijpr.html

DOI-Prefix: https://dx.doi.org/10.16965/ijpr

\section{Article Information}

Received: 21 Dec 2020

Peer Review: 22 Dec 2020

Revised: None
Accepted: 28 Jan 2021

Published (O): 11 Feb 2021

Published (P): 11 Feb 2021

\section{INTRODUCTION}

In rehabilitation following surgical interventions and medical diseases, it is desirable to improve physical function and muscle strength. To assess such changes in muscle performance, accurate, reliable, and applicable methods to evaluate muscle performance should be applied. Furthermore, to define objective goals for and evaluate a rehabilitation program normative data which include information about the influence of variables such as sex, age, height, body mass, and daily physical activity on muscle performance are required [1].

It is also well known that low and declining muscle strength is associated with increased 
mortality, independent of physical activity and muscle mass [2]. It has been well reported that there is significant loss in muscle strength with aging $[3,4]$. Aging is associated with alterations in body composition; there is an increase in body fat percentage and a concomitant decline in lean body mass [5]. Aging, therefore, results in substantial alterations in body composition, with a marked reduction in skeletal muscle mass.

Females have a 3-8 times greater risk for lower extremity injury than similarly trained males [6]. There are generally 3 types of theories to explain these differences in female and male knee injury rates: anatomical, neuromuscular and hormonal. One anatomical theory is related to the quadriceps angle or Q-angle. It has been argued that differences in pelvic structure and lower extremity alignment may account for differences in male and female injury rates [7]. The second theory to explain the gender difference in knee injury incidence relates to neuromuscular differences between males and females [8]. Haycock and Gillette [9] reported similar overall injury rates for male and female collegiate athletes, although they did indicate that females had higher rates of injury involving the patella and joints, including the knee. They speculated that any differences were due to differing levels of training and coaching and not to anatomical or physiological differences, though they presented little evidence to support this conclusion. A third theory relates to the effects of the female hormones estrogen, progesterone and relaxin on the female neuromuscular and musculoskeletal systems. Several reports attribute injury rate differences to estrogen-related differences such as increased joint laxity among women [10]. The contradictory reports of increased laxity could result from the fluctuating effects of estrogen and relaxin on ligament collagen. Evidence suggests that female sex hormones may have significant effects on the female neuromuscular system. Estrogen is known to both directly and indirectly affect the female neuromuscular system [11].

So early identification of the BMI and muscle weakness, can be promoted for developing future rehabilitation by giving proper training in athletes to reduce chance of injuries especially in female athletes. There-for the purpose of this study was to determine the correlation between BMI and hip muscle strength in young female athletes.

\section{METHODS}

Subjects and Methods: This pilot study was conducted among college level female athletes from different colleges of Mangalore. Ethical clearance was obtained from Institutional Ethical Committee. The subjects participating in this study were given patient information sheet containing the study details of and also the inform consent was obtained from the subject prior to the study. 20 college level female athletes between $18-25$ years with free from injury and involved at least $2 \mathrm{hrs}$. per week training session were included in this study. Athletes were excluded if participant had an acute injury during previous six months, had musculoskeletal surgery within the past year.

\section{BMI}

BMI was calculated using the formula:

Weight $(\mathrm{kg}) /[\text { height }(\mathrm{m})]^{2}=\mathrm{BMI}\left(\mathrm{kg} / \mathrm{m}^{2}\right)$

\section{Isometric Hip muscle strength test}

Hip abductor strength: For the hip abductors, participants lay on their left or right side with their knees and hips flexed to $45^{\circ}$ on the examination table. No other pelvic stabilization was provided. The investigator placed resistance on the lateral aspect of the distal femur as the participants tried to move their hip into abduction against the handheld push-pull dynamometer.

Hip flexor strength: For the hip flexors, participants sat on the edge of the examination table with their legs hanging and their hands holding the sides of the table. The investigator placed resistance on the anterior aspect of the distal femur as the participants tried to flex their hip against the handheld push-pull dynamometer.

Hip external rotator strength: For the hip external rotators, participants sat on the table with their hands holding the side and a towel between their thighs. The investigator placed 
resistance on the medial surface of the distal tibia as the participants tried to externally rotate their hip and move their foot towards the other leg against the handheld push-pull dynamometer.

Hip extensor strength: For the hip extensors, participants lay prone with their hips off the table and feet on the ground with the test knee flexed to 90 degrees and the hip relaxed. The investigator applied resistance to the posterior mid portion of the femur as participants tried to extend their hip towards the ceiling against the hand held push-pull dynamometer [12].

Statistical Analysis: Data was analysed using statistical program for social science (SPSS) 22. Results were expressed as mean, standard deviation and frequency. Spearman's correlation coefficient was used to find the relationship between $\mathrm{BMI}$ and hip muscle strength in young female athletes. Level of significance was set as $p<0.05$.

\section{RESULTS}

The total number of 20 young female athletes aged between 18- 25 were included in this study.

\section{Descriptive Statistics of Demographic data}

Table 1: Descriptive statistics of Demographic data.

\begin{tabular}{lccccc}
\hline & N & Minimum & Maximum & Mean & Std. Deviation \\
\hline Age & 56 & 18 & 25 & 20.5 & 2.09762 \\
Height & 56 & 148 & 160 & 153.625 & 2.79976 \\
Weight & 56 & 45 & 58 & 50.875 & 3.83791 \\
BMI & 56 & 19.7 & 24 & 21.5304 & 1.22429 \\
\hline
\end{tabular}

\section{DISCUSSION}

This pilot study was done to determine the relationship between $\mathrm{BMI}$ and hip muscle strength in collegiate female athletes. In this study 20 samples were included who were trained $2 \mathrm{hrs}$ per week with an age group between 18 to 25 years. The athletes were recruited from various colleges in South India. An informed consent was obtained from each participant. Each participant underwent two major procedures of the study ie, BMI was assessed\& Hydraulic handheld push-pull dynamometer to assess the hip muscle strength. Participant height \& weight was taken to calculate the BMI. For measuring hip

\section{Descriptive Statistics of Outcome measures}

Table 2: Descriptive statistics of outcome measures.

\begin{tabular}{ccccc}
\hline & Minimum & Maximum & Mean & Std. Deviation \\
\hline RFS & 3.5 & 6.5 & 5.3036 & 0.67203 \\
\hline LFS & 4 & 7 & 5.2768 & 0.61732 \\
\hline RAS & 4 & 9 & 5.5357 & 1.02184 \\
\hline LAS & 4 & 6 & 4.9107 & 0.61131 \\
\hline RES & 4 & 6 & 4.6964 & 0.62289 \\
\hline LES & 4 & 6 & 4.8571 & 0.43395 \\
\hline RERS & 2.5 & 5 & 4.0625 & 0.68796 \\
\hline LERS & 2.5 & 5 & 3.8571 & 0.65167 \\
\hline
\end{tabular}

\section{Correlation between right hip muscle strength}

\section{and BMI}

Table 3: In this table $r$ value for RFS is -.077 and $p$ value is .571 so it is not statistically significant. For RAS ( $r=-$ $.135) \&(p=.321)$ which shows it is not statistically significant. Similarly, for RES $(r=-.033) \&(p=.808)$ so it is not statistically significant. Then for RERS $(r=-.147)$ $\&(p=.278)$ which shows it is not statistically significant.

\begin{tabular}{|c|c|c|c|c|c|c|}
\hline & & & RFS & RAS & RES & RERS \\
\hline \multirow{2}{*}{$\begin{array}{l}\text { Spearman } \\
\text { correlation }\end{array}$} & \multirow[t]{2}{*}{ BMI } & $\begin{array}{l}\text { Correlation } \\
\text { Coefficient }\end{array}$ & 77 & -0.135 & -0.033 & -0.147 \\
\hline & & $P$ value & 0.571 & 0.321 & 0.808 & 0.278 \\
\hline
\end{tabular}

Correlation between left hip muscle strength and BMI

Table 4: In this table $r$ value for LFS is -.018 and $p$ value is .839 which shows it is not statistically significant. Similarly, for LAS $(r=-.092) \&(p=.500)$ so it is not statistically significant. For LES $(r=-.028) \&(p=.840)$ so it is not statistically significant. Then for LERS $(r=.106)$ $\&(p=.438)$ which shows it is not statistically significant.

\begin{tabular}{|c|c|c|c|c|c|c|}
\hline & & & LFS & LAS & LES & LERS \\
\hline \multirow{2}{*}{$\begin{array}{l}\text { Spearman } \\
\text { Correlation }\end{array}$} & \multirow{2}{*}{ BMI } & $\begin{array}{l}\text { Correlation } \\
\text { Coefficient }\end{array}$ & -0.018 & -0.092 & -0.028 & 0.106 \\
\hline & & P value & 0.893 & 0.5 & 0.84 & 0.438 \\
\hline
\end{tabular}

muscle strength an equipment called 'hydraulic push-pull dynamometer' was used. The isometric strength of four groups of hip muscles were analysed, those are hip flexors, hip extensors, hip abductors and hip external rotators.

In the present study correlation between BMI and hip muscle strength was RFS ( $r=-.077)$, RES $(r=-.033)$, RAS ( $r=-.135)$, RERS ( $r=-.147)$ were not statistically significant with $p$ value $>0.05$. Similarly, Correlation of BMI with left hip flexors was LFS ( $r=-.018)$, LES $(r=-.028)$, LAS $(r=-.092)$, LERS $(r=.106)$ which shows these were not statistically significant with $p$ value $>0.05$.

Nobuyuki Miyatake et al. (2012) conducted a study on 'Relationship between muscle 
strength and anthropometric, body composition parameters in Japanese adolescent'.Leg strength was significantly correlated with height, body weight, hip circumference and lean body mass $(r=0.708)$. However, leg strength per body weight was negatively correlated with body mass index (BMI) [13]. Potential limitations still remain in this study. First, this study was a cross-sectional and not a longitudinal study. Second, 48 men and 189 women in our study voluntarily underwent measurements: they were therefore more likely to be health-conscious compared with the average person.

Noha Abdel Kader Abdel Kader Hasan et al. (2016) has done a study on 'Relation between body mass index percentile and muscle strength and endurance'. The study shows that the BMI had a positive correlation with the muscle strength of quadriceps, triceps, and abdominal muscles while a negative correlation with the endurance time of these muscles [14].

Prasetiowati et al. (2017) has done a study on 'Effect of Body Mass Index on Postural Balance and Muscle Strength in Children Aged 8-10 years'and the result shows that there were no significant differences in hip extensor muscle strength between obese children with overweight and normal weight children $(p=0.527)$. The absolute knee extensor muscle strength in obese group was significantly higher than the over-weight and normal group $(p=0.003)$. However, the relative muscle strength of lower limb for obese children was significantly lower than for normal weight [15]. Markku Kankaanpaa et al. (1998) reported that the paraspinal muscles, as well as the hip extensor muscles, biceps femoris, and gluteus maximus, showed clear decreases in MF (muscle fatigue) during the isometric endurance test in women. MF decrease was highly related to endurance time and $\mathrm{BMI}$ in women [16].

KengoKimozaki et al. (2018) reported that greater BMI and hip abductor muscle strength were independent risk factors for noncontact $\mathrm{ACL}$ injuries in female Japanese high school basketball players. Although performing complete screens may be difficult, attention should be given to $A C L$ injuries, particularly in highly competitive players with strong muscles [17].

Limitations: No proper medical health screening was done before the study and this may lead to an error to the outcome. Other than BMI no anthropometric measurements was analyzed.

\section{CONCLUSION}

Present study concluded that there was no relationship between $\mathrm{BMI}$ and hip muscle strength. Identifying the relationship between $\mathrm{BMI}$ and hip muscle strength may help to prevent lower extremity injury risk in female athletes and also specific muscle group training can be given as rehabilitation protocol.

\section{ABBREVIATIONS USED}

BMI - Body Mass Index

$R-$ Right

$L-$ Left

FS - Flexor strength

ES - Extensor strength

AS - Abductor strength

ERS - External rotator strength

\section{ACKNOWLEDGEMENTS}

My sincere and heart felt gratitude to all the participants, my guide, departmental staff, my parents, my colleagues and friends for their cooperation during the study and preparation of the manuscript.

\section{Conflicts of interest: None}

\section{REFERENCES}

[1]. Harbo, T., Brincks, J., \& Andersen, H. Maximal isokinetic and isometric muscle strength of major muscle groups related to age, body mass, height, and sex in 178 healthy subjects. European journal of applied physiology, 2012;112(1):267-275.

[2]. Metter, E. J., Talbot, L. A., Schrager, M., \& Conwit, R. Skeletal muscle strength as a predictor of all-cause mortality in healthy men. The Journals of Gerontology Series A: Biological Sciences and Medical Sciences 2002;57(10):B359-B365.

[3]. Larsson, L., \& Karlsson, J. Isometric and dynamic endurance as a function of age and skeletal muscle characteristics. Acta Physiologica Scandinavica, 1978;104(2): 129-136.

[4]. Young, A., Stokes, M., \& Crowe, M. Size and strength of the quadriceps muscles of old and young women. European journal of clinical investigation, $1984 ; 14(4): 282-287$. 
[5]. Rogers, M. A., \& Evans, W. J. Changes in skeletal muscle with aging: effects of exercise training. Exercise and sport sciences reviews, 1993;21(1):65-102.

[6]. Smith, H. C., Vacek, P., Johnson, R. J., Slauterbeck, J. R., Hashemi, J., Shultz, S., \& Beynnon, B. D. Risk factors for anterior cruciate ligament injury: a review of the literature-part 1: neuromuscular and anatomic risk. Sports health, 2012;4(1):69-78.

[7]. Zelisko, J. A., Noble, H. B., \& Porter, M. A comparison of men's and women's professional basketball injuries. The American journal of sports medicine, 1982;10(5):297-299.

[8]. Hewett, T. E., Lindenfeld, T. N., Riccobene, J. V., \& Noyes, F. R. The effect of neuromuscular training on the incidence of knee injury in female athletes. The American journal of sports medicine, 1999;27(6):699-706.

[9]. Haycock, C. E., \& Gillette, J. V. Susceptibility of women athletes to injury: myths vs reality. Jama, 1976;236(2):163-165.

[10]. Chandy, T. A., \& Grana, W. A. Secondary school athletic injury in boys and girls: a three-year comparison. Journal of Pediatric Orthopaedics, 1985;5(5):629.

[11]. Hewett, T. E. Neuromuscular and hormonal factors associated with knee injuries in female athletes. Sports medicine, 2000;29(5):313-327.

[12]. Ambegaonkar, J. P., Mettinger, L. M., Caswell, S. V., Burtt, A., \& Cortes, N. Relationships between core endurance, hip strength, and balance in collegiate female athletes. International journal of sports physical therapy, 2014;9(5):604.
[13]. Nobuyuki, M., Motohiko, M., Izumi, T., Noriko, S., Tomohiro, H., \& Takeyuki, N. Relationship between muscle strength and anthropometric, body composition parameters in Japanese adolescents. Health, 2012.

[14]. Hasan, N. A. K. A. K., Kamal, H. M., \& Hussein, Z. A. Relation between body mass index percentile and muscle strength and endurance. Egyptian Journal of Medical Human Genetics, 2016;17(4):367-372.

[15]. Prasetiowati, L., Kusumaningtyas, S., \& Tamin, T. Z. Effect of Body Mass Index on Postural Balance and Muscle Strength in Children Aged 8-10 years. Journal of Krishna Institute of Medical Sciences (JKIMSU), 2017;6(2).

[16]. Kankaanpää, M., Laaksonen, D., Taimela, S., Kokko, S. M., Airaksinen, O., \& Hänninen, O. Age, sex, and body mass index as determinants of back and hip extensor fatigue in the isometric Sørensen back endurance test. Archives of physical medicine and rehabilitation, 1998;79(9):1069-1075.

[17]. Shimozaki, K., Nakase, J., Takata, Y., Shima, Y., Kitaoka, K., \& Tsuchiya, H. Greater body mass index and hip abduction muscle strength predict noncontact anterior cruciate ligament injury in female Japanese high school basketball players. Knee Surgery, Sports Traumatology, Arthroscopy, 2018;26(10):3004-3011.
How to cite this article:
Akshaya M V, Abhilash P V, Priya S. Relationship between BMI and hip
muscle strength in young female athletes to prevent lower extremity injury
risk: A pilot study. Int J Physiother Res 2021;9(1):3754-3758. DOI: 10.16965/
ijpr.2020.190 